\title{
Neurophotonics
}

\section{Coregistering functional near-infrared spectroscopy with underlying cortical areas in infants}

Sarah Lloyd-Fox

John E. Richards

Anna Blasi

Declan G. M. Murphy

Clare E. Elwell

Mark H. Johnson 


\title{
Coregistering functional near-infrared spectroscopy with underlying cortical areas in infants
}

\author{
Sarah Lloyd-Fox, ${ }^{\mathrm{a}, \star}$ John E. Richards, ${ }^{\mathrm{b}}$ Anna Blasi, ${ }^{\mathrm{a}}$ Declan G. M. Murphy, ${ }^{\mathrm{c}}$ Clare E. Elwell, ${ }^{\mathrm{d}}$ and \\ Mark H. Johnson ${ }^{a}$ \\ aBirkbeck, University of London, Centre for Brain and Cognitive Development, Malet Street, London WC1E 7HX, United Kingdom \\ bUniversity of South Carolina, Institute for Mind and Brain, Department of Psychology, Columbia, South Carolina 29208, United States \\ 'Institute of Psychiatry, Sackler Institute for Translational Neurodevelopment, King's College London, London SE5 8AF, United Kingdom \\ dUniversity College London, Department of Medical Physics and Bioengineering, Malet Place Engineering Building, \\ Gower Street, London WC1E 6BT, United Kingdom
}

\begin{abstract}
Functional near-infrared spectroscopy (fNIRS) is becoming a popular tool in developmental neuroscience for mapping functional localized brain responses. However, as it cannot provide information about underlying anatomy, researchers have begun to conduct spatial registration of fNIRS channels to cortical anatomy in adults. The current work investigated this issue with infants by coregistering fNIRS and magnetic resonance imaging (MRI) data from 55 individuals. Our findings suggest that fNIRS channels can be reliably registered with regions in the frontal and temporal cortex of infants from 4 to 7 months of age. Although some macro-anatomical regions are difficult to consistently define, others are more stable and fNIRS channels on an age-appropriate MRI template are often consistent with individual infant MRIs. We have generated a standardized scalp surface map of fNIRS channel locators to reliably locate cortical regions for fNIRS developmental researchers. This new map can be used to identify the inferior frontal gyrus, superior temporal sulcus (STS) region [which includes the superior and middle temporal gyri (MTG) nearest to the STS], and MTG and temporal-parietal regions in 4- to 7-month-old infants. Future work will model data for the whole head, taking into account the properties of light transport in tissue, and expanding to different ages across development. $\odot$ The Authors. Published by SPIE under a Creative Commons Attribution 3.0 Unported License. Distribution or reproduction of this work in whole or in part requires full attribution of the original publication, including its DOI. [DOI: 10.1117/1.NPh.1.2.025006]
\end{abstract}

Keywords: functional near-infrared spectroscopy; magnetic resonance imaging; coregistration; infancy; development.

Paper 14007RR received Feb. 18, 2014; revised manuscript received Aug. 6, 2014; accepted for publication Aug. 11, 2014; published online Sep. 8, 2014.

\section{Introduction}

The development of the new technology NIRS for the study of functional brain imaging in infants has been rapid over the last 15 years, and has been a welcome addition to the very limited choice of methods suitable for use in awake infants. Many believe that functional NIRS (fNIRS) provides an essential bridge between our current understanding of cortical activity in the developing brain, and our knowledge of adult human brain function. Much of what we have learned about infant development has come from behavioral studies, which use looking time paradigms, and the number of developmental cognitive neuroscience studies still remains low. For many years, the primary choice for functional imaging in awake infants has been electroencephalography (EEG), a noninvasive technique with high temporal resolution but relatively poor spatial resolution. A major advantage of fNIRS compared with EEG is that it is less susceptible to data corruption by movement artifacts. For example, EEG data can be corrupted by movements, such as whole body movement, blinks and horizontal eye movements and, therefore, usually requires a large number of trials so that the signal can be averaged. ${ }^{1}$ In contrast, fNIRS data are less affected by whole body movements when the headgear is well-designed, ${ }^{2}$ largely unaffected by eye movements, involves the analysis of a far lower number of trials and has

*Address all correspondence to: Sarah Lloyd-Fox, E-mail: s.fox@bbk.ac.uk the potential to provide individual measures of brain function., Furthermore, it offers more highly spatially resolved images of activation which allows the localization of brain responses to specific cortical regions. Moreover, fNIRS systems are relatively inexpensive and portable, can accommodate a degree of movement from the infants enabling them to sit upright on a parent's lap, and can reveal spatially localized patterns of hemodynamic changes allowing comparisons with functional magnetic resonance imaging (fMRI) data of adult human brain function (for review see Refs. 2, 5, and 6).

With this optical technique, the light migrates from sources to detectors located on the head by traveling through the skin, skull, and underlying brain tissue. ${ }^{7,8}$ In infants, the majority of the light, measured by the detector in each channel (sourcedetector pair), has interrogated cortex approximately midway between source and detector and half this distance in depth from the scalp surface. ${ }^{9}$ The attenuation (or loss) of this light (in the wavelength range 650 to $1000 \mathrm{~nm}$ ) is due to both absorption and scattering effects within these tissues, which will differ according to the age of the participant. Furthermore, the light transport properties of tissue, and the thickness of the tissue and skull differ over development and as a consequence light travels further, and will interrogate more of the brain, in younger infants. ${ }^{9,10}$ Blood oxyhemoglobin $\left(\mathrm{HbO}_{2}\right)$ and deoxyhemoglobin $(\mathrm{HHb})$ chromophores have different absorption properties of near-infrared light enabling blood oxygenation to be measured. If scattering is assumed to be constant, the measured changes in the attenuation of the near-infrared light can be 
used to calculate the changes in blood oxyhemoglobin $\left(\mathrm{HbO}_{2}\right)$, deoxyhemoglobin $(\mathrm{HHb})$, and total hemoglobin $\left(\mathrm{HbT}=\mathrm{HbO}_{2}+\mathrm{HHb}\right)$ in the illuminated tissue. The changes in concentration of these chromophores can be used as surrogate markers of brain-blood oxygen level, and hence provide a means of investigating brain function. Stimulus onset and neuronal activation induce an increase in the concentration of $\mathrm{HbO}_{2}$, which is usually accompanied by a lesser decrease in $\mathrm{HHb}$ concentration. This activation induced vascular response is known as the hemodynamic response function (HRF). The shape of the HRF may vary according to the evoking stimuli (i.e., differences in amplitude are observed between brief and prolonged stimulus presentation) as well as the underlying neural activity.

We anticipate that further refinement and application of fNIRS over the next 10 years will significantly contribute to the advancement of our understanding of the developing brain. Recent work in various research labs has already led to major progress in these areas. For example, the development of multiple source-detector distance arrays to investigate depth discrimination of the hemodynamic response; an ever-increasing number of channels allowing for a wider coverage of the head; and advances in the design of the headgear providing improved quality of the optical signals (for review see Ref. 2). Furthermore, the number of research laboratories that have recently acquired, or are in the process of acquiring, a system for fNIRS is increasing rapidly. However, even for experienced fNIRS researchers, a major challenge remains: there is no capacity for measuring brain structure for anatomical reference of the functional response.

fNIRS measurements are made from sensors on the surface of the scalp making it difficult to establish the exact spatial origin within the brain of the measured hemodynamic response. In fNIRS and EEG studies, it is standard practice to use the 10/20 system $^{11}$ to standardize regions of the scalp for sensor placement using external landmarks. These coordinates have been colocalized with underlying anatomy in infants and adults; ${ }^{12-14}$ however, this method generally provides information about the broad underlying organization of the brain rather than specific cortical regions. Furthermore, the coordinates used in adults do not always overlie the same position in infants, for example, in infants the inferior frontal electrode lies inferior to the frontal lobe as opposed to over that area in adults. ${ }^{12}$ Hence, developmental fNIRS research can benefit greatly from the accurate information provided by structural brain imaging techniques such as MRI. One might assume that we should prefer fMRI for infant research when a localized cortical response is expected. However, though it has been used successfully for some infant research (i.e., Refs. 15 and 16), fMRI is expensive, noisy, and requires the participant to remain very still, usually swaddled or restrained. For this reason, fMRI has generally been restricted to the study of auditory stimuli in sleeping, sedated or newborn infants. The combination of fNIRS functional paradigms and structural MRI data acquired during sleep could, therefore, contribute crucial information about functional cortical responses in awake infants, and the relative effects of changes in head shape, brain size, and morphology in the early growth stages.

The traditional methods for placement of fNIRS channels (midpoint between a source and detector optode) fall into two streams. Head-based fiducial locations-i.e., the 10 to 20 recording system or the position of external landmarks such as the ear/nasion-are measured on individual participant heads and used to align the fNIRS optodes appropriately for each individual. ${ }^{17,18}$ fNIRS optodes are generally housed either in a flexible headgear (such as an EasyCap or elasticized network that stretches to the head size and thus increases the separation distance between source and detector), or within a structured array (with fixed distance between source and detector) secured within a semiflexible headband (i.e., the front of the headband may have a fixed distance between the glabella and optodes, while the headband on the back of the head is adjustable; see Fig. 1). Commonly, for both forms of headgear fNIRS researchers use a range of differently sized caps or headbands for different age groups. Measurements are then taken in relation to head size and external landmarks such as the nasion/ear for each individual. ${ }^{19}$ The first type of design-based on a proportional 10 to 20 system-makes the assumptions that the cortical regions in the developing brain do not change location in relation to external landmarks or proportional changes in head size as the individual ages, and that it does not matter whether the source-detector distances, and, therefore, the depth of measurement differ across infants. Generally, the

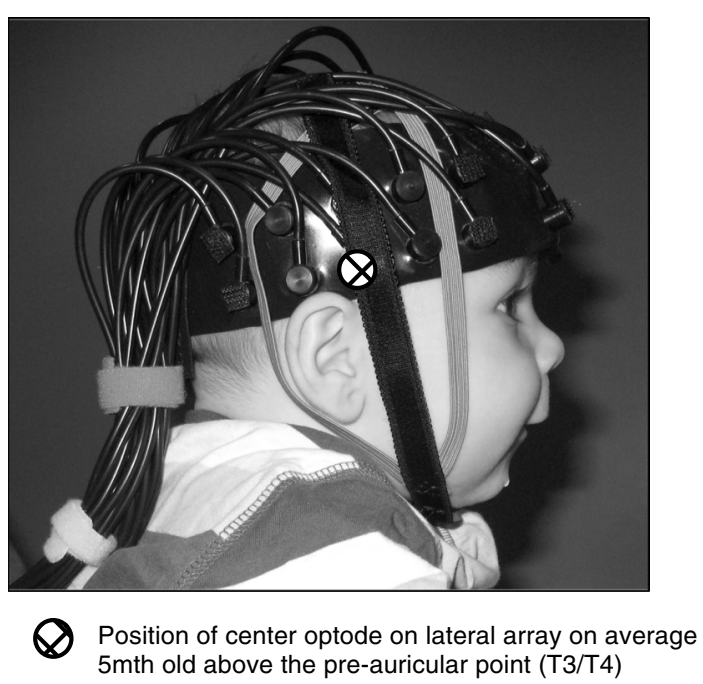

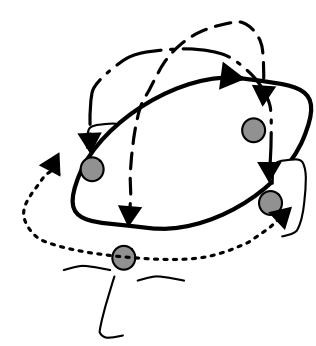
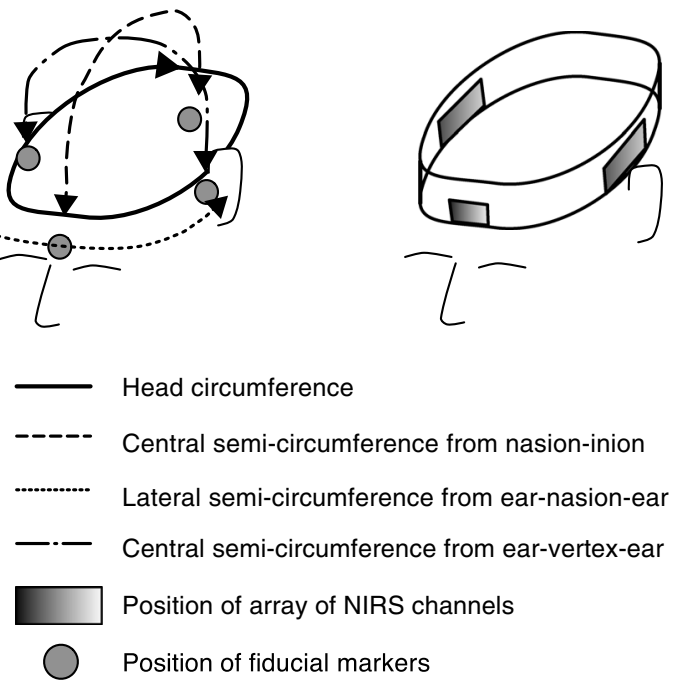

Fig. 1 Functional near-infrared spectroscopy (fNIRS) headgear on an average 5-month-old head, the head measurements taken during the study and the position of the fiducials on the head. 
cap is changed according to head size within one age group, thus the source-detector distances may vary across infants. The second type of design — based on a fixed source-detector distance system-ensures that the source-detector distances are equal across infants, and the depth of measurement is approximately equal, but requires the headband to be available in different sizes for different ages so that the position of the array aligns correctly with the area of interest. Generally, the same headband is used for a range of infants within one age group, thus the array may shift anterior-posterior depending on the size of the infant's head. Therefore, the two systems will result in two scales of differential positioning of optodes on the head across participants. Neither approach places the optodes in analytically identified locations on the scalp; some type of external measurement is necessary to quantify channel location. A typical procedure in adults is to use a device to locate the channels in three-dimensional (3-D) space on the participant's head, e.g., photogrammetry (pictures around the head in 3-D space) or a radio frequency localizer (e.g., Polhemus Fasttrack digitizer, Colchester, Vermont). However, these methods are currently largely unsuitable for awake infants as they either require the infant to be motionless, or they take too much time to setup and administer and are, therefore, currently unsuitable for infant participants. Instead developmental researchers can routinely record: (1) photographs of the head from several angles (front, sides, top, and back) and (2) measurements of the infant's head circumference, distance between glabella, ears, vertex, preauricular points and inion, and the location of the channels and arrays relative to these anatomical landmarks.

In recent work at the Centre for Brain and Cognitive Development (CBCD), we have developed a database of infants who underwent both an MRI structural scan and took part in an fNIRS study. We investigated the efficacy of fNIRS channel placement to anatomical regions of interest (ROIs) by coregistering scalp locations with MRI volumes within the same individual infants (for similar work in adults see Ref. 20). Technical challenges include identification of the channel locations in space, registration between the fNIRS channel space and the MRI space, and correct placement of the fNIRS sources and detectors on the MRI volume. Recently, researchers have begun to coregister fNIRS channels to standard brain space (i.e., Montreal Neurological Institute-MNI) using probabilistic registration methods to identify associated anatomical regions. ${ }^{21-23}$ However, there is a paucity of research that directly compares fNIRS and MRI data within individuals to confirm the reliability of this approach. Coregistration of individual fNIRSMRI data has been largely restricted to adult work, ${ }^{20}$ or with infants using adult standard brain space (see Ref. 24 though Ref. 25 for fNIRS-MRI coregistration on a single infant). Therefore, there is a great need for studies to be undertaken with multiple infant MRIs to assess the impact of individual variations and age-dependent variations on cortical surface structures relative to anatomical landmarks. This work could establish whether future fNIRS infant studies could use a referential age-appropriate map of underlying cortical regions relative to scalp fNIRS coordinates rather than acquiring participant-specific MRIs which would be high cost and unsuitable in many populations.

Several pediatric anatomical atlases are now available from MRIs taken over the first years of life. ${ }^{26,27}$ Recently, for instance, Phillip et al. ${ }^{28-30}$ developed methods for creating stereotaxic atlases for individuals and for age-specific MRI templates at
$3,4.5,6,7.5,9$, and 12 months of age. First, based on procedures developed for 2-year old participants, ${ }^{27}$ they generated a stereotaxic atlas based on the LONI Probabilistic Brain Atlas project (56 manually delineated areas for 40 participants; LPBA $40^{31}$ ) and one based on the Hammers adult brain atlas (83 manually delineated areas for 30 participants; Hammers atlases $\left.;^{32-34}\right)$. This was done for each participant by registering the 40 manually segmented adult MRIs in their native space (used to create the LPBA40) to each participant, or the 30 manually segmented adult MRIs from the Hammers atlas, and creating an individual stereotaxic atlas for each participant. They found a very good correspondence between areas generated in the automatic atlas and areas coming from manually segmented lobar areas. ${ }^{29,30}$ Second, they created a manually segmented lobar atlas for each of the age-specific MRI templates. This atlas was transformed to individual participants and compared against manually segmented lobar areas in individual participants. A good correspondence was found between the transformed atlas and the manually segmented areas. ${ }^{28-30}$ We, therefore, adopted their procedure for generating individual participant LPBA40 atlases and so produced anatomical labels for our individual infant MRIs. We also used the appropriate age-specific manual lobar atlas from the average MRI template and transformed it by linear registration techniques to each individual infant MRI.

There is a paucity of research that directly compares fNIRS and MRI data within individuals, and coregistration of individual fNIRS-MRI data has been largely restricted to adult work, ${ }^{20}$ or with infants using adult standard brain space (see Ref. 24 though Ref. 25 for fNIRS-MRI coregistration on a single infant). Hence, our study sought to answer several questions. How similar are the coregistered anatomical locations of the fNIRS channels-in the CBCD-designed headgear-across a group of 4- to 7-month-old infants? Can we use individual infant coregistered fNIRS-MRI data to identify nearest neighbor fNIRS channel scalp surface locations for cortical ROIs? How do changes in head size and age of infant affect the reliability of the coregistration of fNIRS channels with underlying anatomy? Can the use of an age-specific average MRI atlas give adequate information of fNIRS-MRI registration for a given level of spatial resolution? Finally, can we generate a standardized scalp surface map of fNIRS channel locators relative to cortical ROIs for developmental fNIRS researchers for whom individual infant MRIs are not available?

\section{Methods}

\subsection{Participants}

The participants were retrospectively selected from a study conducted at the CBCD at Birkbeck, University of London (Session 1) and the Institute of Psychiatry (IoP) at King's College London (Session 2). They were selected because they had both an MRI structural scan (undertaken at the IoP) and had participated in an fNIRS study at CBCD. For the majority of the infants, these sessions were within 3 weeks of each other (median-5 days): 1 infant had the two sessions at 24 days apart (all analyses were also run with this infant excluded, but results did not change so they were retained in the sample). Most participants $[N=55 ; 23$ female; mean age 150.07 (SD 21.44 ), range 119 to 201 days] were recruited from the CBCD participant database and were healthy 4- to 7-monthold infants. The infants were from a varied ethnic and 
socioeconomic background, predominantly white European (75\%), black European (mixed white/African/African Caribbean) or Asian European (mixed white/South Asian/Far Eastern Asian). ${ }^{35}$ Twenty-two of the infants were in an atrisk for autism cohort, but this group did not differ significantly in head circumference and no gross structural abnormalities were observed. All parents gave their written informed consent. The Birkbeck, University of London and Institute of Psychiatry and South London and Maudsley research ethics committees approved the study. A fuller description of the participants is given elsewhere. ${ }^{3,15,36}$

\subsection{Procedure}

A fuller description of the methods for each session is given in the previous work. ${ }^{3,15,36}$

During Session 1 the infants participated in an fNIRS study at $\mathrm{CBCD}$ using the UCL-fNIRS system ${ }^{37}$ and custom built CBCD-fNIRS headgear (used in previously published work $^{3,4,19}$ ). The CBCD fNIRS headgear houses three arrays to form up to 33 channels. The two lateral arrays contain 10 optodes each (five sources and five detectors) to form 26 channels (2-cm distance). The frontal array has six optodes (three sources and three detectors) to form six channels (two at $2 \mathrm{~cm}$, four at $2.5-\mathrm{cm}$ distance). The headgear consists of a fixed length headband with the three arrays (frontal, left lateral, and right lateral) attached within this headband in fixed positions. The frontal array is positioned so that the midpoint of the array is aligned with the center of the forehead, directly above the glabella (midpoint between the eyebrows). The lateral arrays are fixed so that they are positioned with the middle optode (third furthest from anterior) over the tragus (see Fig. 1) on an average 5-month-old infant. The distance from the glabella to this optode on the headband is constant across participants-11 cm back from the glabella-as this was found to be the average distance in this age range (measured on $300+$ infants at CBCD). The position of the source and detector optodes can be calculated for each infant as they are held within fixed arrays, which are contained within a fixed length of headband (from the glabella to arrays). The headgear is placed on the head so that the midpoint of the headband is aligned with the glabella, and the lateral arrays sit on the scalp where the top of the ear joins to the head (therefore, the midpoint of the middle optode is $1 \mathrm{~cm}$ above the join between the top of the ear and head). The position of the fNIRS headgear on the head was recorded in the following steps. The head circumference $(\mathrm{H}-\mathrm{C})$, the lateral semicircumference (LSC) from ear to glabella to ear, the central semicircumference from nasion to inion, and the semicircumference from ear to vertex to ear were measured. After the infant was fitted with the fNIRS headgear, photographs were taken from the front and to the left and right. These pictures recorded the positioning of the fNIRS arrays and headgear relative to the nasion, ears, and other fiducials.

During Session 2 infants participated in an MRI study at the IoP. ${ }^{15}$ All scanning sessions were scheduled around each infant's nap time. Upon arrival, the families were situated in a quiet room where the infant would fall asleep on the MRI examination bed. When asleep, the infant was swaddled, the MedVac Vacuum Immobilization Bag (CFI Medical Solutions, Fenton, Michigan) was fastened and sound attenuation devices were attached (Natus MiniMuffs Noise Attenuators and MR-compatible piezoelectric headphones, San Carlos,
California; MR confron GmbH, Magdeburg, Germany). MRI data were acquired on a GE 1.5 T Twinspeed MRI scanner (General Electric, Milwaukee, Wisconsin). A T2 weighted fast spin echo (FSE) dataset was acquired $(256 \times 168$ rectangular matrix, 2-mm slice thickness, $0-\mathrm{mm}$ slice gap, field of view $=18 \mathrm{~cm}, \mathrm{TR}=4500, \mathrm{TE}=113 \mathrm{~ms}$, echo train length $=17$ ). The FSE images were reviewed by a pediatric neuroradiologist to screen for any abnormalities. Gradient rise times were limited in order to reduce the noise of the pulse sequences to approximately $70 \mathrm{~dB}$. Daily quality assurance was carried out to ensure high signal-to-ghost ratio, high signal-to-noise ratio and excellent temporal stability using an automated quality control procedure. ${ }^{38}$ The body coil was used for RF transmission and an 8-channel head coil for RF reception. ${ }^{38}$ An experimenter and a parent stood in the scanner room to observe the infant's behavior at all time and the infant's heart rate was monitored using a pulse oxymeter secured on the toe. The session ended if the infant awoke and showed discomfort.

\subsection{Data Processing and Analysis}

\subsubsection{MRI average templates}

For each participant, we identified the MRI average template that was closest in age to the participants (at the time of the MRI session), and the participant was assigned to either a 4.5- or 6-month group. This resulted in 36 infants in the 4.5month group [mean age-137.25 days (119 to 157 days); mean $\mathrm{H}-\mathrm{C}-41.6 \mathrm{~cm}(38.5$ to $44.8 \mathrm{~cm})]$ and 19 infants in the 6-month group [mean age-174.4 days (160 to 201 days); mean $\mathrm{H}-\mathrm{C}-42.1 \mathrm{~cm}(38.3$ to $44.3 \mathrm{~cm})]$.

Average MRI templates constructed from the USC-MCBI MRI volumes were used (for details of MRI data acquisition see Refs. 39 and 40; see available online in Ref. 41). The scans had $1 \mathrm{~mm}^{3}$ resolution and a sufficient field of view to cover from the top of the head down to the neck. The templates were constructed with an iterative procedure. ${ }^{39-43}$ This procedure created a tentative average volume from the individual participants of a specific age, registered individual participants' MRIs to the tentative average volume, transformed the individual volumes in size and orientation with nonlinear registration (using ANTS, 44 "Advanced Normalization Tools"), and then reconstructed the average from the transformed files. For the present study, we used only the templates created from the 3.0 T MCBI MRIs. The average MRI templates had associated stereotaxic atlas MRI volumes (lobar, LPBA40).

\subsubsection{File preparation, scalp and skull volume, stereotaxic atlases}

The individual MRI volumes and average MRI templates were prepared for analyses in three steps. First, the brain was extracted from the whole-head MRI volume using procedures adapted from the brain extraction tools of FSL. ${ }^{45-47}$ Because the participant MRIs in this study were T2-weighted scan, the brain extraction procedure was supplemented by also identifying the cerebrospinal fluid (CSF) in the T2W volume by thresholding the voxel values to identify the CSF, and then removing the CSF from the extracted brain to identify the non-CSF aspects in the brain volume. Second, the scalp surface was identified with the betsurf procedure. ${ }^{45}$ This program uses a brain mesh with voxel values around the brain to establish 
approximate masking levels for the skull and scalp surfaces, and provides an MRI volume mask of the outer surface of the scalp. Third, a scalp surface volume was constructed from the scalp mask by eroding the scalp mask by $2 \mathrm{~mm}$ and using the difference between the original mask and the eroded volume. The same procedure was used to construct the cortical surface.

Two stereotaxic atlases were constructed for each participant MRI. These stereotaxic atlases consisted of an MRI volume that identifies anatomical areas and can be used for automatic MRI procedures to identify anatomical locations on the brain extracted from the MRI volume. The first atlas was a macro-anatomical atlas constructed on each individual participant MRI using the LONI Probabilistic Brain Atlas (LPBA40; ${ }^{31}$ which is based on manual delineation of 40 adults resampled into common space). We adopted the adult-brain atlas for the infant MRIs as it has been shown that the relative macro-structural anatomical pattern of the infant cortex is similar to adults. ${ }^{48}$ This was done by an established procedure (for details of the use of this method for 2-year-olds see Ref. 27 and for infants see Refs. 28-30) that used the 40 adult-manually segmented brains from the LPBA40 atlas and registered these brains to an individual infant participant, transforming the adult volume to the individual infant's MRI space. Then, we used a majorityvote fusion procedure, which combines the 40 labeled MRI adult volumes to each individual infant to identify a macro-anatomical area for each brain voxel in the individual infant MRI volume (following methods used in Refs. 26 and 27). This fusion-based approach has been shown to be more efficient than direct warping. ${ }^{33,49}$ This procedure also results in a good correspondence between the automatically generated atlas and manually segmented lobar areas. ${ }^{28-30}$ The second atlas was a lobar atlas that identified the major cerebral lobes, some sublobar areas, and subcortical areas. ${ }^{28-30}$ This atlas was constructed by manual segmentation of the major lobes on average MRI templates in infants. The individual participant MRI was linearly registered to the age-appropriate average MRI template, and the age-appropriate lobar atlas was transformed by the linear registration matrix into the MRI space. This approach has been shown to result in a good correspondence between the transformed atlas and manually segmented lobar areas. ${ }^{28-30}$ The procedures for generating macro-anatomical and lobar atlases have also been recently used across a range of individual infant MRIs and age-appropriate templates from 3 to 12 months of age. ${ }^{28-30}$ Figure 2 shows a 6-month-old age-appropriate MRI template $^{40}$ and the lobar and LPBA40 atlases overlaid on this template.

\subsubsection{Scalp surface locations projection to cortex and cortical areas for projections}

A series of fiducial measurements were made on each of the individual infants' T2W MRI volume. ${ }^{50}$ First, the anterior commissure (AC) was manually identified in the image. The location of the AC was defined in meter coordinates and was used as the 3 -D origin of the MRI volume. ${ }^{51}$ The AC location was marked on a 3-D rendered volume using MRIcron ${ }^{52}$ by placing a $2-\mathrm{mm}$ spherical mask on the location. ${ }^{53}$ Preauricular points were also defined at the anterior roots of the tragi on MRI slices in addition to the nasion (a dent at the upper root of the nose bridge) and the inion (an external occipital proturberance). ${ }^{54}$ In infants, these scalp markers are used in lieu of external markers (such as vitamin-E capsules or digitized channel recordings) so they should be detectable both on the participant's head from the photographs and on his/her MRI. ${ }^{20}$ Then, the position of the fNIRS channels relative to these scalp landmarks were recorded for each infant using the following procedure. The head measurements and photographs of the infants wearing the fNIRS arrays and headgear (see earlier section for further details) were used to identify four fiducial landmarks on the 3-D-rendered MRI volume of the infant head (for similar work coregistering EEG electrodes see Ref. 53). Fiducials were placed as spherical masks (i) on the MRI volume at the front of the headgear over a centered marker on the headband which was aligned with the nasion, (ii) on the right and left sides at the lowermiddle optode location which on an average 4- to 6-monthold infant are aligned with the preauricular points (or T3 and
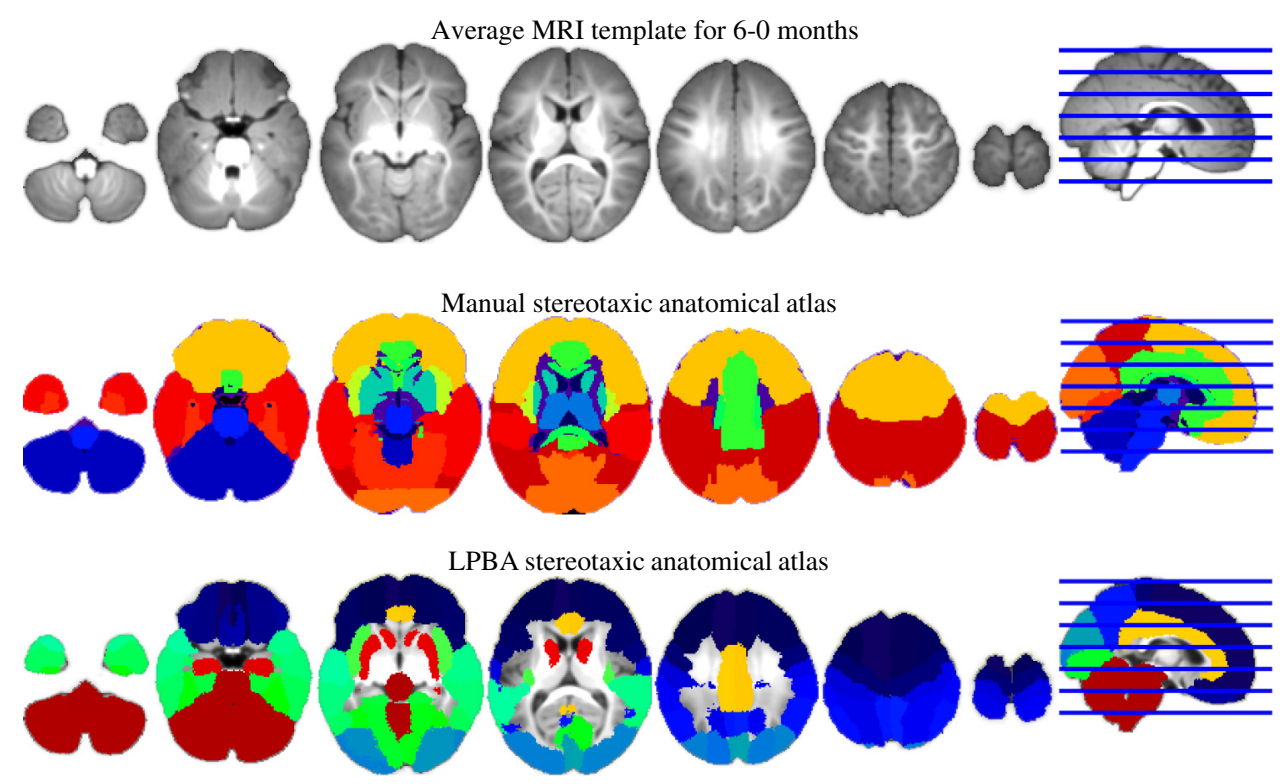

Fig. 2 A 6-month-old age-appropriate magnetic resonance imaging (MRI) template ${ }^{40}$ and the lobar and LPBA40 atlases overlaid on this template (adapted from Ref. 28). 


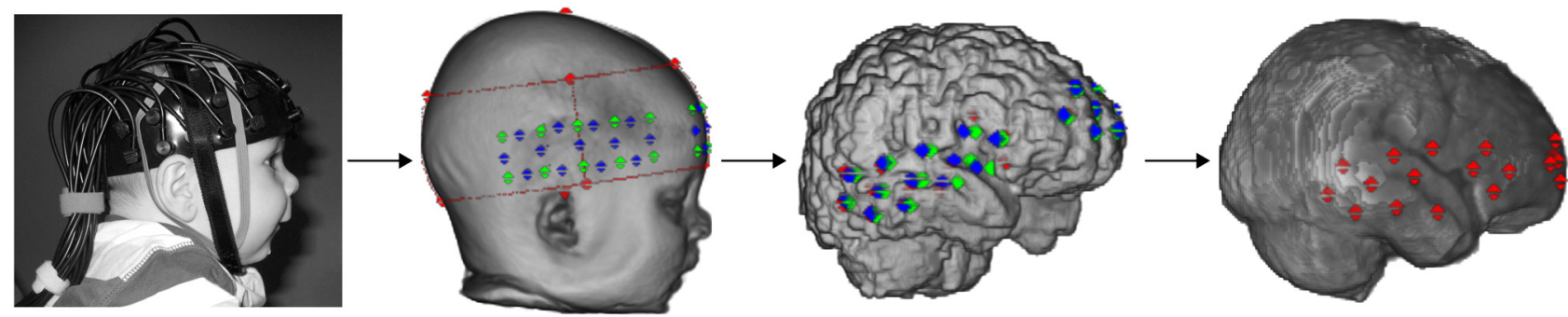

Fig. 3 A step-by-step guide to coregistration of scalp surface fNIRS channels with underlying anatomy. Photos and measurements taken of the head-fiducial markers for the holder, optode, and channel locations are identified on the scalp surface volume from their MRI; the channel locations are projected down from the scalp surface volume to the cortical surface volume; and the channel locations can also be projected onto an age-appropriate average template.

T4: 10 to 20 system), and (iii) over the rear at the location where the bottom of the headband met the medial location on the head. To verify these positions, they were compared against the head measurements of each infant (from the 3-D MRI volume) and the known dimensions of the fNIRS headgear. For example, on the fNIRS headgear used in this study the centered marker on the forehead is at a fixed distance of $11 \mathrm{~cm}$ from the lowermiddle optode on each lateral array-which is positioned above the ear. This measurement was crosschecked on the MRI volume with the location identified from the photographs. Once these fiducials were identified, software was used to construct the fNIRS headgear on the MRI volume from these locations and the known dimensions of the headgear in relation to these (see Fig. 3). Optode locations were defined using the position of the fiducials and the known rigid geometry of the sensor arrays. Finally, channel locations were defined as occurring on the scalp midway between optode source/detector locations. Once the channels were located on the head, the 3-D coordinates of the fiducial relative to the $\mathrm{AC}$ were recorded. As a final step, we referred back to the photographs of the individual infants to check whether the headgear was placed correctly, although it should be aligned with the glabella and ears along the referential axial curve, though human error during placement may have caused misalignment. Reliability of fNIRS headgear placement was recorded for each infant to inform later analyses. A recent methodological paper ${ }^{25}$ used similar procedures to identify fiducials of known anatomical landmarks on one 12-month-old infant MRI volume to project 10 to 20 coordinates and then a virtual fNIRS holder onto the surface of the head and coregister with macro-anatomical positions from the LPBA40 atlas (as used in the current work).

The scalp location for each channel was projected from the scalp surface volume to the cortical surface volume using the "balloon" method. ${ }^{55}$ This method expands the area in the scalp surface volume around a scalp location until the expansion reaches the nearest point on the cortical surface volume ("balloon" expansion). The cortical areas for each projection point on the cortical surface were used to identify the closest segmented area in the two stereotaxic atlases. Each projected location on the cortical surface volume was used in a look-up procedure to find the closest lobar or macro-anatomical site in the stereotaxic atlases. For the LPBA40 atlas, each location on the cortical surface corresponded to a voxel on the extracted brain volume, and each voxel of the brain volume was identified in the stereotaxic atlas. Figure 3 shows a step-by-step flowchart of the processes described in this section: photo of a single infant; the scalp surface volume from their MRI with fiducial markers for the
Table 1 Atlas locations of the functional near-infrared spectroscopy (fNIRS) channels across the group of infants that were assigned to (a) the 4.5-month-old age-appropriate template and (b) the 6-month-old age-appropriate template. The label of the channel is followed by (\%) of infants with this region. Regions are reported when the number of allocated infants $\geq 20 \%$ of the group. The italicized labels are those with a differing majority lobar or macro-anatomical region between the two groups of infants (4.5- versus 6-month-olds). Note that the groups match if you allow any channel label where the group scores $\geq 20 \%$ (i.e., channel 9: 4.5 months-superior temporal gyrus $44 \%$, postcentral gyrus $36 \%$; 6 months-superior temporal gyrus $37 \%$, postcentral gyrus $53 \%$ ).

fNIRS

channels Lobar atlas Macro-anatomical atlas (LPBA40)

(a) Infants with the 4.5 month old template

Left lateral fNIRS array

1 Frontal (89) Inferior frontal gyrus (78)

2 Frontal (100) Inferior frontal gyrus (100)

3 Frontal (100) Inferior frontal gyrus (92)

$4 \quad$ Frontal (86) Inferior frontal gyrus (69)

5 Temporal (81) Superior temporal gyrus (67)

$6 \quad$ Frontal (79) Inferior frontal gyrus (39), precentral gyrus (36)

$7 \quad$ Temporal (61), Superior temporal gyrus (64) frontal (36)

8 Temporal (100) Middle temporal gyrus (72), superior temporal gyrus (22)

$9 \quad$ Parietal (47) Superior temporal gyrus (44), temporal (31) postcentral gyrus (33)

Frontal (22)

Superior temporal gyrus (61), middle temporal gyrus (36)

Temporal (100) Middle temporal gyrus (83)

12 Temporal (86)

Superior temporal gyrus (64), middle temporal gyrus (25)

13 Temporal (88) Middle temporal gyrus (83) 
Table 1 (Continued).

\begin{tabular}{l} 
fNIRS \\
channels Lobar atlas $\quad$ Macro-anatomical atlas (LPBA40) \\
\hline
\end{tabular}

\section{Right lateral fNIRS array}$$
15
$$

\section{Frontal fNIRS array}

27

Frontal (100)

Frontal (100)

Frontal (100)

Frontal (100)

Frontal (100)

Frontal (100)

Inferior frontal gyrus (69)

Inferior frontal gyrus (92)

Inferior frontal gyrus (75)

Inferior frontal gyrus (64)

Superior temporal gyrus (44), middle temporal gyrus (25), inferior frontal gyrus (24)

Inferior frontal gyrus (42), precentral gyrus (33)

Superior temporal gyrus (53), precentral gyrus (25)

Middle temporal gyrus (64), superior temporal gyrus (33)

Superior temporal gyrus (42), postcentral gyrus (31)

Superior temporal gyrus (53), middle temporal gyrus (44) temporal gyrus (42)

Superior temporal gyrus (50), middle temporal gyrus (31)

Middle temporal gyrus (50)

Superior frontal gyrus (92)

Middle frontal gyrus (78), superior frontal gyrus (22)

Middle frontal gyrus (94)

Superior frontal gyrus (94)

Middle frontal gyrus (100)

Superior frontal gyrus (81)

Middle frontal gyrus (56),

Table 1 (Continued).

\begin{tabular}{|c|c|c|}
\hline $\begin{array}{l}\text { fNIRS } \\
\text { channels }\end{array}$ & Lobar atlas & Macro-anatomical atlas (LPBA40) \\
\hline 8 & Temporal (100) & $\begin{array}{l}\text { Middle temporal gyrus (74), superior } \\
\text { temporal gyrus }(26)\end{array}$ \\
\hline 9 & $\begin{array}{l}\text { Parietal (63), } \\
\text { frontal }(21)\end{array}$ & $\begin{array}{l}\text { Postcentral gyrus (53), superior } \\
\text { temporal gyrus (37) }\end{array}$ \\
\hline 10 & Temporal (100) & $\begin{array}{l}\text { Superior temporal gyrus ( } 79) \text {, middle } \\
\text { temporal gyrus }(21)\end{array}$ \\
\hline 11 & Temporal (100) & Middle temporal gyrus (90) \\
\hline 12 & Temporal (90) & Superior temporal gyrus (84) \\
\hline 13 & Temporal (100) & $\begin{array}{l}\text { Middle temporal gyrus (68), superior } \\
\text { temporal gyrus }(21)\end{array}$ \\
\hline
\end{tabular}

Right lateral fNIRS array

14

Frontal (95)

Inferior frontal gyrus (74), lateral orbitofrontal gyrus (21)

Frontal (100)

Inferior frontal gyrus (100)

Frontal (100)

Inferior frontal gyrus (79), middle frontal gyrus (21)

superior frontal gyrus (44)

(b) Infants with the 6-month-old template

\section{Left lateral fNIRS array}

1

2

3

4

4

Temporal (68)
frontal (32)

$6 \quad$ Frontal (95)

$7 \quad$ Temporal (74), frontal (26)
Inferior frontal gyrus (90)

Inferior frontal gyrus (100)

Inferior frontal gyrus (100)

Inferior frontal gyrus (90)

Superior temporal gyrus (63), inferior frontal gyrus (26)

Inferior frontal gyrus (53), precentral gyrus (42)

Superior temporal gyrus (68), precentral gyrus (26)
$17 \quad$ Frontal (95)

18 Temporal (68), frontal (32)

$19 \quad$ Frontal (95)

Frontal (53), temporal (47)

Inferior frontal gyrus (95)

Superior temporal gyrus (63), inferior frontal gyrus (26)

Inferior frontal gyrus (58), precentral gyrus (37)

Superior temporal gyrus (42), precentral gyrus (26), inferior frontal gyrus (21)

Temporal (100) Middle temporal gyrus (58), superior temporal gyrus (42)

Parietal (53), $\quad$ Postcentral gyrus (42), superior frontal (26) temporal gyrus (32), preceontral Temporal (21) gyrus (21)

Superior temporal gyrus (79), middle temporal gyrus (21)

Temporal (100) Middle temporal gyrus (95)

Superior temporal gyrus (68) parietal (21)

Middle temporal gyrus (74), superior temporal gyrus (21)

\section{Frontal fNIRS array}

$27 \quad$ Frontal (100)

Superior frontal gyrus (84)

Frontal (100)

Superior frontal gyrus (68), middle frontal gyrus (32)

Frontal (100)

Middle frontal gyrus (95)

Frontal (100)

Superior frontal gyrus (100)

Middle frontal gyrus (100) frontal gyrus (42)
Frontal (100) Middle frontal gyrus (63), superior frontal gyrus (37) 
fNIRS headgear, optode, and channel locations; the cortical surface volume with the channel locations projected down from the scalp surface volume; and the channel locations on an ageappropriate average template.

\section{Results}

\subsection{Comparison of the Coregistered MRI Anatomical Regions and the Location of the fNIRS Channels}

For each infant, we collated data on the position of the fNIRS channel using the two stereotaxic atlases outlined in Sec. 2. Table 1 lists the fNIRS channels and the corresponding lobar areas from the lobar atlas, and macro-anatomical locations from the LPBA40 atlas for the 55 infants. The proportion of infants with a particular channel lying over a particular cortical region is given in brackets (\%). It can be seen that differing channels could be described as having "stable" (over $80 \%$ of group) and "unstable" locations in the lobar atlas. The unstable locations occur in channels that sit near the lateral sulcus between frontal, temporal, and parietal regions (see Fig. 4). These are reflected in the variability seen in the macro-anatomical atlas.

The fNIRS channels were also projected onto the 4.5- and 6month average MRI templates using the positioning method and fixed measurements from the CBCD-headgear (i.e., middle lateral optode $11 \mathrm{~cm}$ from glabella). Table 2 outlines the channels and corresponding macro-anatomical locations (from the LPBA40 atlas). As with the individual infant MRI-fNIRS data, there are some unstable channels over the lateral sulcus which are found between the frontal and temporal lobes where the anatomical location of the channel shifts across the younger and older templates. However, the difference in the coregistration of fNIRS channels and anatomy between the 4.5- and 6-month template is minimal (only three channels have differing anatomical labels in each of the lateral frontal-temporal fNIRS arrays).

\subsection{Identifying Nearest Neighbor fNIRS Channel Scalp Surface Locations Over Regions of Anatomical Interest}

This dataset of MRI and fNIRS data on each individual infant allows us to identify target regions of anatomical interest and assess the variability of the position of nearest neighbor fNIRS channels across infants. The two lateral CBCD arrays are designed to be positioned on the scalp surface over frontal and temporal cortical regions. Therefore, we undertook an analysis on three cortical surface ROIs within the social brain network: the inferior frontal gyrus (IFG), the superior temporal sulcus (STS), and the temporoparietal junction (TPJ).

For each infant, the positions of the ROIs were identified on their own MRI either by registering an age-appropriate MRI ROI to each infant's TPJ or by using the participant's own atlas defined using the lobar, LPBA40 and Hammer atlases (IFG, STS). The TPJ was defined as the posterior superior temporal and middle temporal gyrus bordering with the inferior supramarginal and angular gyrus, ${ }^{4}$ in accord with the broad area identified in a meta-analysis of research in adults by Overwalle and Baeten. ${ }^{56}$ The three cortical surface ROIs and the location of the channels in the left and right hemispheres on the cortical surface volume are illustrated in Fig. 5. Following this, the minimum distance between the closest part of each ROI and each channel location on the cortical surface volume (projected down from the scalp surface volume) was calculated for each infant. The results for the three nearest channels for each ROI in each hemisphere are given in Table 3 for the group of infants, and in Table 4 for the 4.5- and 6-monthold MRI average templates. For the age-appropriate templates, the location of the six nearest neighbor fNIRS channels on the cortical surface volume were directly over the IFG for both the 4.5 and 6 month versions, and ranged from 4 to $14.3 \mathrm{~mm}$ for the TPJ and from 0 to $3.31 \mathrm{~mm}$ for the STS. The range of values was similar across the two templates. For the group of infants, the median distance from the cortical ROIs to the nearest neighbor fNIRS channels ranged from 2.37 to $3.37 \mathrm{~mm}$ for the IFG ROI,
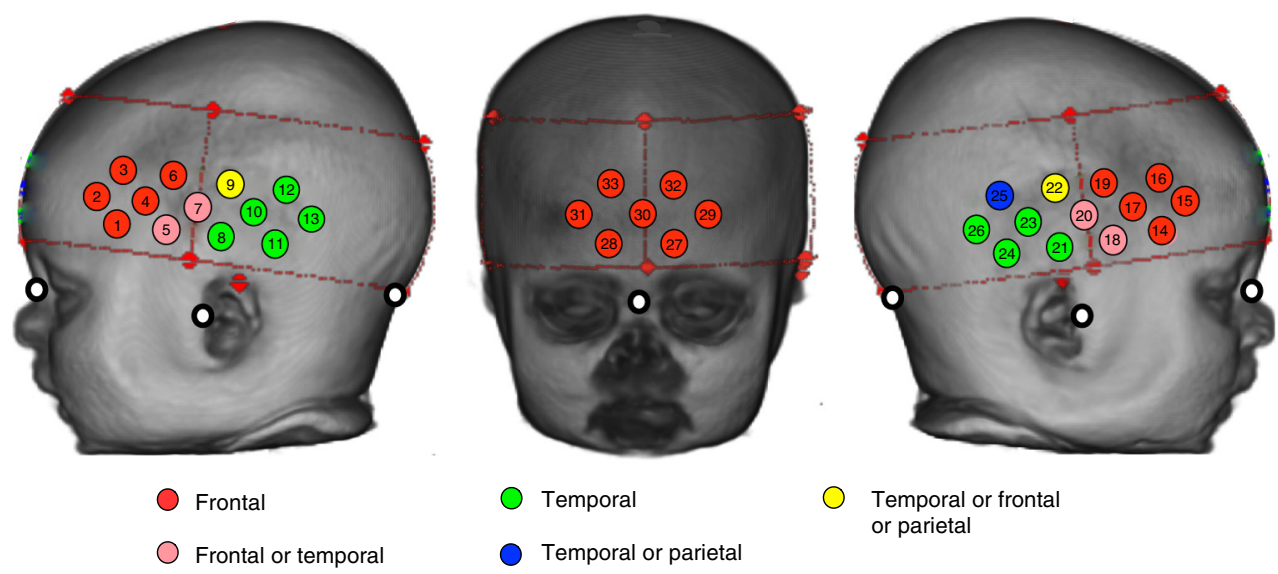

Fig. 4 The fNIRS channels are projected onto a three-dimensional reconstruction of an infant. The red dashed lines and markers identify the position of the fNIRS headband on the infant head. For each fNIRS channel located within this headband, the identity of the underlying lobe (using the lobar atlas) is illustrated according to whether or not-when the channel was projected onto the cortical surface-over $75 \%$ of the group had a common region (frontal/temporal) or whether or not the identified region was split across the group with $30 \%$ to $60 \%$ in each of two to three lobes. The white markers indicate the position of the nasion, inion, and preauricular points on the infant head. Note that for the infants in the 4.5- and 6month group all channels have the same majority identity accept for channel 5 and 25 (see Table 1). 
Table 2 Macro-anatomical atlas (LPBA40) regions for the projection of each fNIRS channel onto the cortical surface for the 4.5- and 6month MRI template. The italicized channels are those with a differing macro-anatomical region between the two templates.

\begin{tabular}{lll} 
fNIRS channels & 4.5-month template & 6-month template \\
\hline Left lateral fNIRS array & \\
1 & Inferior frontal gyrus & Inferior frontal gyrus \\
2 & Inferior frontal gyrus & Inferior frontal gyrus \\
3 & Inferior frontal gyrus & Inferior frontal gyrus \\
4 & Superior temporal gyrus & Superior temporal gyrus \\
5 & Middle temporal gyrus & Middle temporal gyrus \\
6 & Superior temporal gyrus & Postcentral gyrus \\
7 & Middle temporal gyrus & Middle temporal gyrus \\
8 & Middle temporal gyrus & Middle temporal gyrus \\
9 & Superior temporal gyrus & Superior temporal gyrus \\
10 & Middle temporal gyrus & Middle temporal gyrus \\
11 & Inferior temporal gyrus & Inferior temporal gyrus \\
12 & Middle temporal gyrus & Middle temporal gyrus \\
13 & Inferior temporal gyrus & Middle temporal gyrus
\end{tabular}

Right lateral fNIRS array

$\begin{array}{lll}14 & \text { Lateral orbitofrontal gyrus } & \text { Inferior frontal gyrus } \\ 15 & \text { Inferior frontal gyrus } & \text { Inferior frontal gyrus } \\ 16 & \text { Inferior frontal gyrus } & \text { Inferior frontal gyrus } \\ 17 & \text { Superior temporal gyrus } & \text { Precentral gyrus } \\ 18 & \text { Middle temporal gyrus } & \text { Middle temporal gyrus } \\ 19 & \text { Precentral gyrus } & \text { Precentral gyrus } \\ 20 & \text { Middle temporal gyrus } & \text { Superior temporal gyrus } \\ 21 & \text { Middle temporal gyrus } & \text { Middle temporal gyrus } \\ 22 & \text { Superior temporal gyrus } & \text { Superior temporal gyrus } \\ 23 & \text { Middle temporal gyrus } & \text { Middle temporal gyrus } \\ 24 & \text { Inferior temporal gyrus } & \text { Inferior temporal gyrus } \\ 25 & \text { Middle temporal gyrus } & \text { Middle temporal gyrus } \\ 26 & \text { Inferior temporal gyrus } & \text { Middle temporal gyrus }\end{array}$

Frontal fNIRS array

27

Superior frontal gyrus

28

Middle frontal gyrus

29

30

Middle frontal gyrus

Superior frontal gyrus

31

Middle frontal gyrus

32

Middle frontal gyrus

33

Middle frontal gyrus
0.71 to $8.46 \mathrm{~mm}$ for the TPJ ROI and 2.01 to $4.6 \mathrm{~mm}$ for the STS ROI. For the IFG ROI, analyses showed that over $80 \%$ of the infants had the six nearest neighbor fNIRS channels positioned at a maximum of $5 \mathrm{~mm}$ from the IFG. For the TPJ ROI, the nearest neighboring fNIRS channel in the left hemisphere was within $2 \mathrm{~mm}$ of the ROI in $84 \%$ of infants, dropping to $58 \%$ in the right hemisphere. The remaining four fNIRS channels (second and third nearest) were on average further away from the TPJ ROI with $65 \%$ of the group of infants within $10 \mathrm{~mm}$ of the ROI. Finally, for the STS ROI $83 \%$ of the infants had the two nearest neighboring fNIRS channels within $6 \mathrm{~mm}$ of the ROI in the left hemisphere, dropping to $68 \%$ in the right hemisphere. The third nearest fNIRS channels were on average further away from the STS ROI with $80 \%$ of the group within $10 \mathrm{~mm}$ of the ROI.

To illustrate these distances relative to the position of the fNIRS channels on the scalp surface volume, for each channel the group median distance from each ROI in relation to the distance of the channel from the glabella is illustrated in Fig. 6. As expected, the channels in the anterior portion of the fNIRS arrays are closest to the IFG while the channels in the posterior portion of the fNIRS arrays are closest to the STS and TPJ.

\subsection{Cross Reliability of fNIRS Channel Locations: Assessing the Effect of Age and Head Size}

The group of infants ranges in age from 119 to 201 days $($ median $=148)$. Head circumference varies between 38.29 and $44.78 \mathrm{~cm}$ (median $=41.75)$, with the LSC from ear via glabella to ear varying between 19.23 and $23.90 \mathrm{~cm}($ median $=21.43)$ (measurements taken from the 3-D reconstructed MRI images of each infant head). The latter two are highly correlated $(r=0.717, p<0.001)$. Age and head circumference are also correlated $(r=0.322, p<0.001)$, though to a lesser degree.

To investigate the reliability of the location of the fNIRS channels over anatomical regions, we conducted a multiple linear regression analysis. The model was designed to examine whether age, head circumference, and LSC were significant predictors of the distance of the channels from each ROI (IFG, TPJ, and STS). Including the LSC allowed us to investigate whether the location of the fNIRS channels in the lateral arrays over underlying cortical regions was related to the position of the ear, as researchers often align fNIRS channels with this external landmark when positioning on the head. For these analyses, we only included the infants with fNIRS headgear precisely aligned with the midpoint of the frontal array directly above the glabella $(N=32$; if headgear was misaligned by more than $0.5 \mathrm{~cm}$ the infant was excluded from these analyses). Using the multiple linear regression enter method, a significant model emerged. Overwhelmingly, age was found to be a significant predictor of distance across the three ROIs. In summary, for the distance to IFG the regression coefficients of 15 channels had a $p$-value of $<0.01$ and a further five had $<0.05$; for TPJ 16 channels had a $p$-value of $<0.01$ and a further $10 \mathrm{had}<0.05$; and for STS two channels had a $p$-value of $<0.01$ and a further 17 had $<0.05$. The majority of the fNIRS channels that were lying directly over or nearest to the ROIs (according to the macro-anatomical atlas; see Fig. 4 and Table 1) were not predicted by age (IFG-channels 1,2,3,4, 14, 15, and 16; TPJ-channels 12, 13, 22, 23, 25, and 26; the pattern is less clear for STS). The variation explained by the linear model (for age as a predictor) ranges from 18.1 to $54.2 \%\left(R^{2}\right)$. Note that when the residuals were plotted the majority of the channels with significant predictors of 


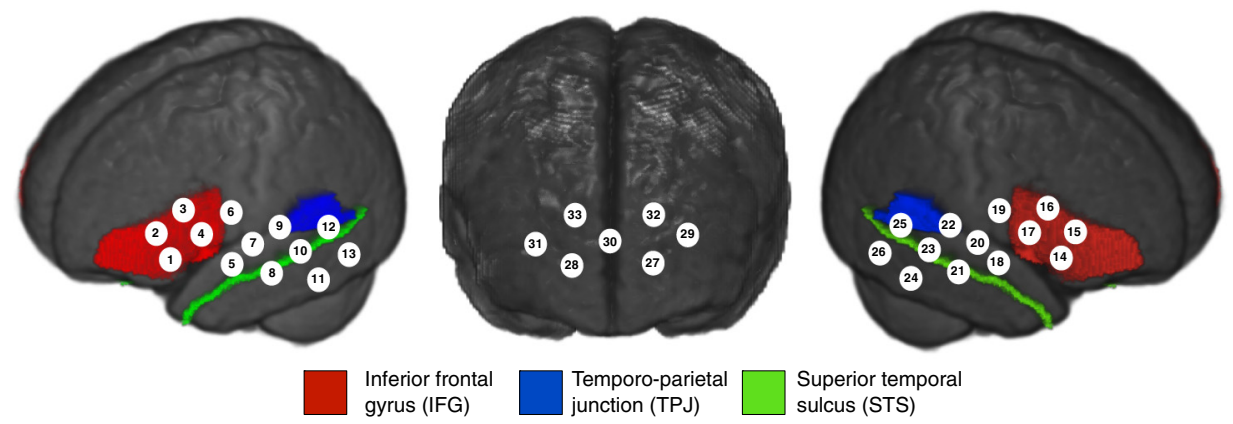

Fig. 5 fNIRS channel locations and the three brain regions used for the regions of interest (ROIs) analysis projected onto the 4.5 -month template.

age displayed normal distribution and constant variance (homoscedasticity). Overall, in the IFG analysis, age is negatively correlated [with the distance between the anatomical ROI on their MRI (IFG) and the location of the fNIRS channel] in channels, which are located over the temporal cortex. For the TPJ analysis, age is positively correlated [with the distance between the anatomical ROI on their MRI (TPJ) and the location of the fNIRS channel] in channels, which, for the majority, are located over frontal and anterior temporal cortex (see Fig. 7 for example plots of the correlations). There were also three channels in the STS analyses that reported head circumference as a significant predictor (and LSC for two of these); however, these results should be treated with caution given that it is unlikely that they would pass a test for multiple comparisons.

\section{Discussion}

fNIRS is a neuroimaging method that can measure localized functional brain responses. However, as fNIRS cannot provide anatomical information, it has been difficult for researchers to claim precision when localizing the response to known brain regions. Recently, researchers have investigated spatial registration of fNIRS channels to cortical anatomy in adults, both for individual MRI-fNIRS data and using standardized MRI space. ${ }^{20}$ However, resources and limitations have thus far prevented this work in infants and children, where developmental change in brain anatomy and function is at its greatest. The current work coregistered fNIRS scalp-surface and MRI anatomical information of infants from 4 to 7 months of age using two

Table 3 The average group distance of the three nearest fNIRS channels to the ROls. Note that the fourth nearest channel for IFG (channels 2 and 15) and STS (channels 12 and 25) in each hemisphere is only $1 \mathrm{~mm}$ (mean and median) further away than the third nearest channels.

\begin{tabular}{|c|c|c|c|c|c|c|}
\hline \multirow[b]{2}{*}{ Region of interest } & \multicolumn{3}{|c|}{ Left hemisphere } & \multicolumn{3}{|c|}{ Right hemisphere } \\
\hline & IFG & TPJ & STS & IFG & TPJ & STS \\
\hline Nearest channel & 4 & 12 & 10 & 17 & 25 & 21 \\
\hline Mean distance from ROI (mm) & 2.7 & 1.85 & 3.21 & 3.34 & 2.65 & 4.09 \\
\hline Median distance from ROI (mm) & 2.37 & 0.71 & 2.01 & 2.91 & 1.17 & 4.08 \\
\hline Minimum distance from ROI (mm) & 0 & 010.1 & 0 & 0 & 0 & 0 \\
\hline Maximum distance from $\mathrm{ROI}(\mathrm{mm})$ & 10.25 & 10.1 & 12.57 & 13.3 & 11.1 & 12.25 \\
\hline Second nearest channel & 1 & 9 & 8 & 14 & 22 & 23 \\
\hline Mean distance from ROI (mm) & 3.01 & 8.5 & 3.37 & 3.37 & 6.51 & 4.53 \\
\hline Median distance from $\mathrm{ROI}(\mathrm{mm})$ & 3.14 & 8.21 & 3 & 3.31 & 6.53 & 4.14 \\
\hline Minimum distance from ROI (mm) & 0 & 0 & 0 & 0 & 0 & 0 \\
\hline Maximum distance from ROI (mm) & 11.79 & 20.7 & 13.3 & 10.72 & 21.4 & 10.3 \\
\hline Third nearest channel & 3 & 10 & 13 & 16 & 23 & 26 \\
\hline Mean distance from ROI (mm) & 3.05 & 8.65 & 5.73 & 3.4 & 8.5 & 5.91 \\
\hline Median distance from ROI (mm) & 3.05 & 8.14 & 4.6 & 3.37 & 8.46 & 4.27 \\
\hline Minimum distance from $\mathrm{ROI}(\mathrm{mm})$ & 0 & 1.22 & 0 & 0 & 0 & 0 \\
\hline Maximum distance from ROI (mm) & 14.8 & 19.1 & 21.63 & 10.72 & 18.58 & 19.03 \\
\hline
\end{tabular}


Table 4 The distance of the three nearest fNIRS channels to the ROls using the 4.5- and 6-month template.

\begin{tabular}{|c|c|c|c|c|c|c|}
\hline \multirow[b]{2}{*}{ Region of interest } & \multicolumn{3}{|c|}{ Left hemisphere } & \multicolumn{3}{|c|}{ Right hemisphere } \\
\hline & $\mathrm{IFG}$ & TPJ & STS & IFG & TPJ & STS \\
\hline \multicolumn{7}{|l|}{ 4.5-month-old MRI template } \\
\hline Nearest channel & 3 & 9 & 7 & 16 & 22 & 18 and 20 \\
\hline Distance from channel (mm) & 0 & 4 & 0 & 0 & 6 & 1 \\
\hline Second nearest channel & 1 & 12 & 9 & 15 & 25 & 22 and 25 \\
\hline Distance from channel (mm) & 0 & 5 & 1.41 & 0 & 8 & 3 \\
\hline Third nearest channel & 2 & 13 & 12 & 14 & 19 & \\
\hline Distance from third nearest channel $(\mathrm{mm})$ & 0 & 14.3 & 2.82 & 0 & 9 & \\
\hline \multicolumn{7}{|l|}{ 6-month-old MRI template } \\
\hline Nearest channel & 3 & 9 & 7 & 14 & 22 & 18 and 20 \\
\hline Distance from channel (mm) & 0 & 6 & 1 & 0 & 4 & 1.41 \\
\hline Second nearest channel & 4 & 12 & 5 & 15 & 25 & 25 \\
\hline Distance from channel (mm) & 0 & 7.07 & 2.23 & 0 & 6.08 & 2.24 \\
\hline Third nearest channel & 1 & 6 & 12 & 16 & 19 & \\
\hline Distance from third nearest channel (mm) & 0 & 13.19 & 3.31 & 0 & 13.64 & \\
\hline
\end{tabular}

anatomical atlases $\left(\operatorname{lobar}^{28}\right.$ and LPBA $\left.40^{31}\right)$. Photos and measurements were taken of the head while the infant took part in an fNIRS study; fiducial markers for the headgear, optode, and channel locations were identified on the scalp surface volume from their MRI; the channel locations were projected down from the scalp surface volume to the cortical surface volume; and the channel locations were projected onto age-appropriate average templates.

In this work, coregistration of fNIRS-MRI demonstrated which CBCD fNIRS channels were positioned over stable regions of the head across the group of 4- to 7-month-old infants. The fNIRS channel position within the lateral arrays could effectively predict the anatomical label from the MRI to a high degree, particularly for lobar labeling. For both the 4.5-month-olds and the 6-month-olds, in 20 out of the 26 channels the lobar atlas label was the same across $\geq 75 \%$ of the group of infants. For the macroanatomical atlas, the number of channels with the same label across $\geq 75 \%$ of the group of infants dropped to seven channels for the 4.5-month-olds and 12 channels for the 6-month-olds. This is not surprising given that the macro-anatomical labels define more precise localized areas within regions while the lobar labels define larger areas of the cortex. From these atlas findings, we can conclude that the channels that are positioned over the lateral sulcus between the frontal and temporal lobes are the most unreliably placed for prediction of underlying anatomy as the likelihood of the channel being in either lobe can drop to a chance level. Furthermore, the precise macro-anatomical atlas labels within the temporal cortex (i.e., the difference between the superior, middle, and inferior temporal gyri) are more difficult to estimate for the 4.5-month-olds than the 6-month-olds, particularly in the right hemisphere. In addition to the individual infant MRI volume coregistration, we also coregistered the position of the CBCD fNIRS channels on the scalp surface with underlying anatomy on the age-appropriate average MRI templates. It was found that the position of the channels in the age-appropriate average templates and those identified in the individual infant MRIs were highly similar for the stable regions of the head described above. These findings suggest that for at least certain regions of the frontal and temporal cortex, fNIRS channels could be reliably placed over ROIs within this age range using age-appropriate templates without the need for individual infant MRIs (though when available, individual MRIs will always give superior resolution as the age and head size of a participant will not always correspond to the average age and head size of the average MRI templates). We should note that these analyses have been done for the CBCD-designed fNIRS headgear using one type of lobar atlas and one type of macro-anatomical atlas. If we extend these analyses to a second macro-anatomical atlas (Hammers ${ }^{32}$ ) then different proportional labeling of regions are found for some channels. For example, rather than the majority of the infants being allocated a definition of IFG for the anterior lateral channels, the registration reports an equal split across the group for IFG and MFG. Further, for the channels which lie over the superior, middle, and inferior temporal gyri according to the LPBA40 atlas, the Hammers atlas defines these according to anterior, central divisions within these gyri, or posterior temporal lobe depending on the position along the anterior-posterior axis. Therefore, while the lobar definitions are more consistent, the macro-anatomical definitions of channel locations may vary according to the atlas used. 
(a)

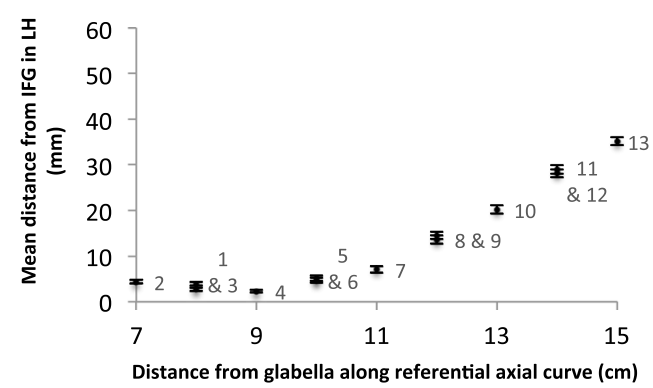

(c)

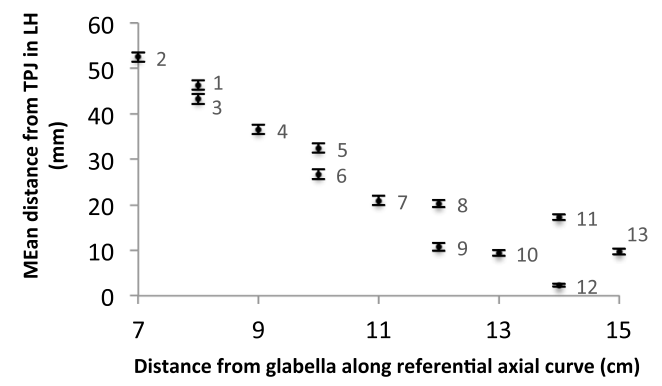

(e)

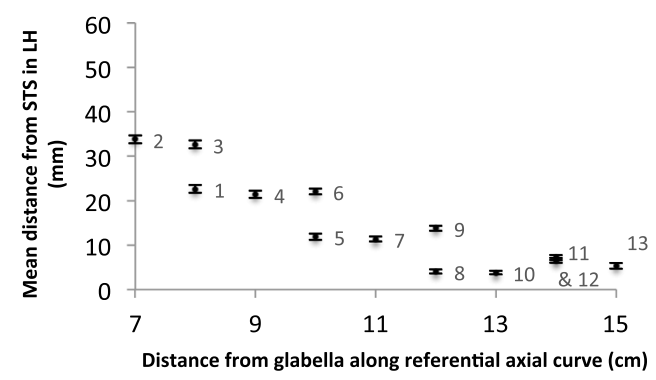

(b)

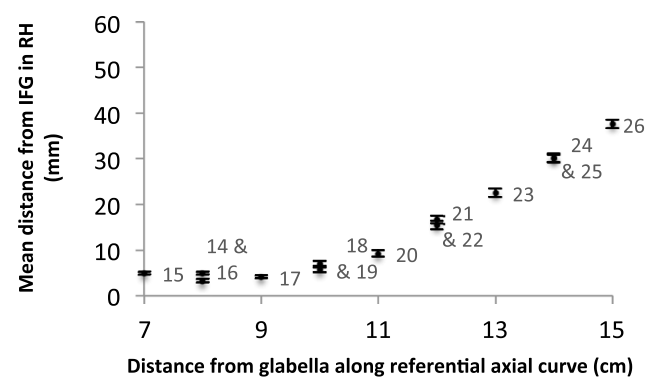

(d)

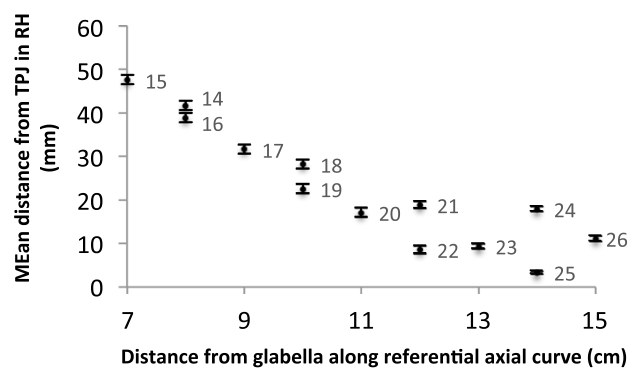

(f)

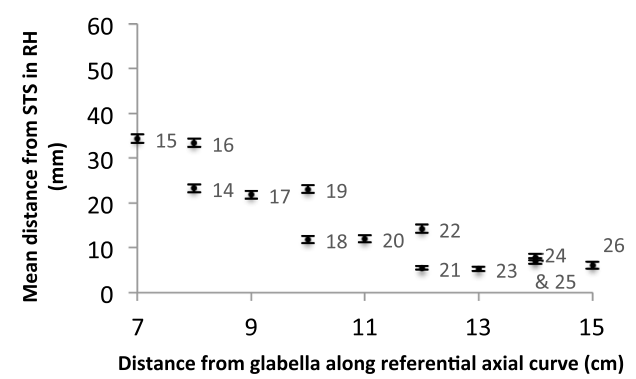

Fig. 6 The median distance of each channel from each ROI (IFG, TPJ, STS) plotted in relation to the distance of the fNIRS channel from the glabella in the axial plane. The channel numbers are labeled on each plot. Standard error bars showing the interindividual differences are included, however as the maximum SE was $1.41 \mathrm{~mm}$, they may not be clear for some data points.

Using the social brain network as a model of interest, we also investigated whether we could identify fNIRS channels which were nearest neighbors to anatomical ROIs of either a gyrus (IFG), a sulcus (STS), or an area between two lobes (TPJ). Overall, the coverage of the CBCD-fNIRS channels allowed measurement of brain responses directly over all of these ROIs for the majority of infants. Furthermore, these broadly corresponded with the nearest neighbor channels identified for the age-appropriate MRI templates. Given that there were some discrepancies between the individual infant data and the MRI templates, we advise the use of two-three nearest neighbor ROI channels when using the age-appropriate templates to calculate the location of target fNIRS channels in situations when corresponding
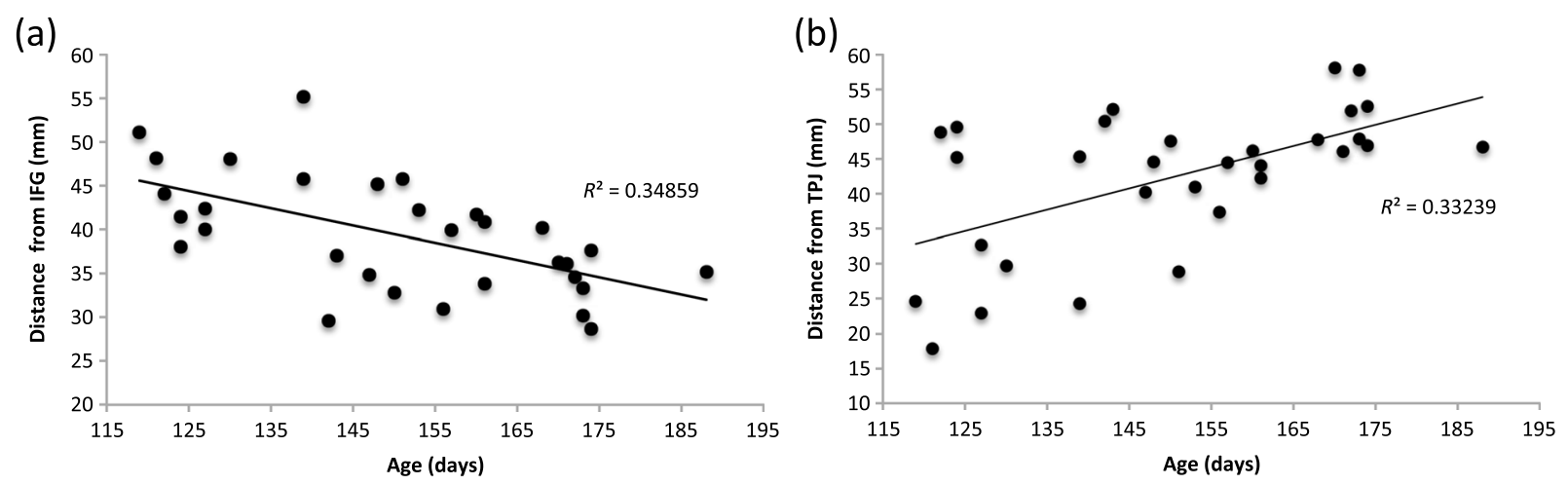

Fig. 7 Correlations of the individual infants' distance between the anatomical ROI on their MRI (IFG or TPJ) and the location of the fNIRS channel on their scalp surface, compared with their age for (a) channel 13 which is situated over the left posterior middle temporal gyrus and (b) channel 14 which is located over the right inferior frontal gyrus. 
individual infant MRIs are not available, as was implemented in a recent work. ${ }^{4}$

Interestingly, the linear regression model suggested that age was the most reliable significant predictor of changes in the distance between the anatomical ROI on individual infants' MRIs and the location of the fNIRS channel on their heads, though note that this predictor only explained between $10 \%$ and $50 \%$ of the variance. Conversely, head circumference and LSC from ear to ear via forehead were not significant predictors, suggesting (i) that changes in head circumference alone (in other words an increase in the circumference of the axial plane), or (ii) the position of the ears relative to the head circumference, did not significantly impact on the location of the fNIRS channels relative to the underlying anatomy across the group of 4- to 7-month-olds. These findings suggest that changes in the head circumference in the axial plane within this age range do not significantly affect scalp surface relations with underlying cortical anatomy, and neither does the position of the ear.

We have generated a standardized scalp surface map of fNIRS channel coordinates for the most stable cortical regions within the frontal and temporal lobes from our infant MRI and fNIRS data. This standardized map could be applied across a range of fNIRS systems by fNIRS developmental researchers interested in these cortical regions and infants in this age
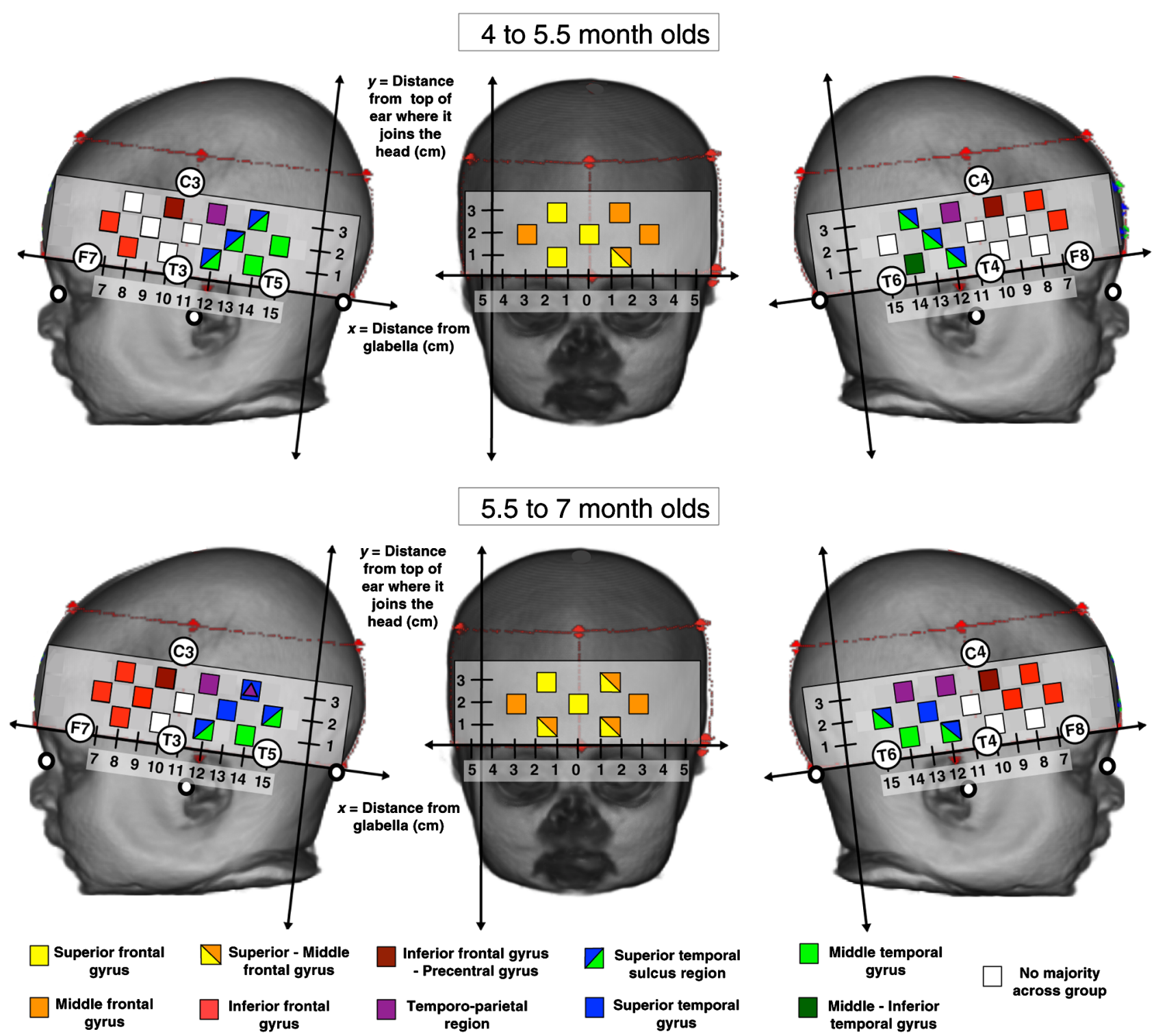

Fig. 8 Reference maps for ROIs in the frontal, temporal, and parietal cortex for placement of fNIRS channels in infants of 4 to 5.5 months (a) and 5.5 to 7 months (b). The regions highlighted were identified during the atlas projections in $75 \%$ to $100 \%$ of the 55 infants tested. The distances given are relative to a referential axial curve between the glabella and the point at which the top of the ear joins the head. Note that for the superior temporal sulcus and temporoparietal locators these are defined as regions as the identity across the group was split between the superior temporal-middle temporal gyri and superior temporal-postcentral gyri respectively (the atlases do not define sulci). The positions with a purple marker are closest/overlapping with the temporoparietal junction (median distance of channel from TPJ is $<2 \mathrm{~mm}$ ). The white markers indicate the position of the nasion, inion, and preauricular points and the red dashed lines and markers identify the position of the fNIRS headband on the infant head. 
range. The set of coordinates provided in Fig. 8 can be used to identify regions of frontal and temporal cortex of infants from approximately 4 to 7 months of age and/or with a head circumference within the range of 39 to $45 \mathrm{~cm}$. The regions provided are the IFG, STS region [which includes the superior and middle temporal gyri (MTG) nearest to the STS], MTG and the temporoparietal region (which includes the STG and parietal cortex nearest to the lateral sulcus). The fNIRS channels that have been identified as being positioned on the scalp surface over these cortical ROIs exhibit a high tolerance of change across our group of infants. Researchers can use the scalp surface coordinates provided in Fig. 8 to target these ROIs for the study of localized group responses, and during the investigation of individual infant responses the channels will be positioned over these target regions for $75 \%$ to $100 \%$ of the infants. This standardized surface map may be utilized by researchers using a wide range of fNIRS headgear and arrays. To calculate appropriate scalp surface locations for fNIRS channel placement in this age group, researchers can measure the distance from the glabella to the fNIRS channels along the referential axial curve (between the glabella and the point at which the top of the ear joins the head) in the headgear that they use with their fNIRS system. If the arrays of sources and detectors are at a fixed distance from the glabella as with the CBCD headgear then this can be measured once and used across the group of infants for one study. If the array of sources and detectors provided with the fNIRS system change with the size of the infants head (i.e., housed within a different EasyCap for each head size, i.e., 39 to $45 \mathrm{~cm}$ ) then reference channels should be measured in relation to the glabella across the range of caps used. With this information researchers can then refer to this standardized map to identify ROIs. Note that the anatomical definitions may vary according to the atlas used, and that these macro-anatomical definitions have been provided by the LPBA40 atlas. ${ }^{31}$ In future work, we could extend our standardized scalp surface map by virtually modeling 10 to 10 positions on each infant MRI. This would allow the investigation of cross-reliability of the anatomical atlas labels for each 10 to 10 position across the group of infants, and provide a set of stable and unstable coordinates for fNIRS channel placement over a range of target regions of the brain. Furthermore, the degree of tolerance of change in both age and head size could be modeled across the whole head rather than just along the axial plane.

\subsection{Limitations of the Current Work}

A major limitation of this study is that we have only investigated these effects within 4- to 7-month-old infants without gross abnormalities in brain development. Future work should extend this to the investigation of MRI-fNIRS data on younger and older infants to examine a wider spectrum of developmental change, although we note that approximately $50 \%$ of published infant fNIRS studies study this age range (i.e., Refs. 2 and 57; also see the Database of Infant functional NIRS studies). ${ }^{58}$ Furthermore, we were limited to conclusions regarding the cortical surface regions of the frontal and temporal lobes. To build on the current findings, models of light transport should be incorporated into the current dataset to provide a more accurate estimate of the penetration of fNIRS light into the infant head. In most fNIRS work, we rely on the approximation that the maximum likelihood of the origin of the light measured by detectors is halfway between the source light and detector pair, and half this distance in depth from the scalp surface (i.e., the majority of light measured by a $20 \mathrm{~mm}$ channel would be from a depth of $10 \mathrm{~mm}$ ). Our study provides a unique dataset of over 50 sets of infant MRI-fNIRS data. Furthermore, the fNIRS arrays in the CBCD configurations allow for measurement of longer separations of source-detector pairs and, therefore, allows investigation of depth related change in functional activation. ${ }^{59,60}$ Therefore, we could use measures from the structural MRIs of skull and CSF thickness variation both within and across infants to inform models of light transport and investigate depth related anatomical ROIs. In future work, we should also consider undertaking a direct comparison of fNIRS headgear that places arrays of fixed source-detector channels over ROIs, in contrast to headgear that expand proportionally with increases in head size (thus source-detector channel sizes change), to see how these factors effect coregistration with underlying anatomy and light transport models.

\section{Conclusions}

The current study sought to answer several questions about the accuracy with which fNIRS headgear channels map onto specific cortical areas. This work crucially underpins the confidence with which conclusions can be drawn about fNIRS cortical activation patterns. We describe how when fNIRS channels are carefully placed in relation to skull landmarks, the identification of underlying anatomy as measured using structural MRI can be obtained from age-appropriate templates and atlases. Further, individual infant coregistered fNIRS-MRI data can be used to identify nearest neighbor fNIRS channel scalp surface locations for cortical ROIs. With our dataset, differences in the age of infants between 4 and 7 months affected the coregistration of fNIRS channels with underlying anatomy more than other variables. In correspondence with this, an age-specific average MRI atlas was as effective for identifying the correct lobe of fNIRS measurement on the surface of the head as implementing the procedure with an individual participant MRI. Finally, we generated a scalp surface map of fNIRS channel locators that maps onto cortical "social brain" ROIs for future studies in which individual infant MRIs are not available.

\section{Acknowledgments}

We are very grateful to the enormous contributions that the families have made to this study. The research was supported by The UK Medical Research Council (G0701484), a grant from The Simons Foundation (No. SFARI201287 to M.H.J.), the BASIS funding consortium led by Autistica (www.basisnetwork.org), the National Institutes of Health (NICHD R37HD18942 to J.E.R.), the Biomedical Research Centre at IoP and the Innovative Medicines Initiative Joint Undertaking under Grant Agreement No. 115300, resources of which are composed of financial contribution from the European Union's Seventh Framework Programme (FP7/2007-2013) and EFPIA companies' in kind contribution.

\section{References}

1. F. Dick et al., "Neuroimaging methods," in Educational Neuroscience, D. Mareschal, B. Butterworth, and A. Tolmie, Eds., pp. 13-45, John Wiley \& Sons, Chichester, UK (2013).

2. S. Lloyd-Fox, A. Blasi, and C. E. Elwell, "Illuminating the developing brain: the past, present and future of functional near infrared spectroscopy," Neurosci. Biobehav. Rev. 34(3), 269-284 (2010). 
3. S. Lloyd-Fox et al., "The emergence of cerebral specialization for the human voice over the first months of life," Soc. Neurosci. 7(3), 317-330 (2012).

4. S. Lloyd-Fox et al., "Cortical activation to action perception is associated with action production abilities in young infants," Cereb. Cortex (2013) (in press).

5. J. Gervain et al., "Near-infrared spectroscopy: a report from the McDonnell infant methodology consortium," Dev. Cogn. Neurosci. 1 (1), 22-46 (2011).

6. Y. Minagawa-Kawai et al., "Optical imaging of infants' neurocognitive development: recent advances and perspectives," Dev. Neurobiol. 68(6), 712-728 (2008)

7. F. F. Jöbsis, "Noninvasive, infrared monitoring of cerebral and myocardial oxygen sufficiency and circulatory parameters," Science 198(4323), 1264-1267 (1977).

8. A. Villringer and B. Chance, "Non-invasive optical spectroscopy and imaging of human brain function," Trends Neurosci. 20(10), 435442 (1997).

9. Y. Fukui, Y. Ajichi, and E. Okada, "Monte Carlo prediction of nearinfrared light propagation in realistic adult and neonatal head models," Appl. Opt. 42(16), 2881-2887 (2003).

10. A. Duncan et al., "Measurement of cranial optical path length as a function of age using phase resolved near infrared spectroscopy," Pediatr. Res. 39(5), 889-894 (1996).

11. H. H. Jasper, "Report of the committee on methods of clinical examination in electroencephalography," Electroencephalogr. Clin. Neurophysiol. 10(2), 370-375 (1958).

12. W. T. Blume, R. C. Buza, and H. Okazaki, "Anatomic correlates of the ten-twenty electrode placement system in infants," Electroencephalogr. Clin. Neurophysiol. 36, 303-307 (1974).

13. R. W. Homan, J. Herman, and P. Purdy, "Cerebral location of international 10-20 system electrode placement," Electroencephalogr. Clin. Neurophysiol. 66(4), 376-382 (1987).

14. M. Okamoto et al., "Three-dimensional probabilistic anatomical craniocerebral correlation via the international 10-20 system oriented for transcranial functional brain mapping," Neuroimage 21(1), 99-111 (2004).

15. A. Blasi et al., "Early specialization for voice and emotion processing in the infant brain," Curr. Biol. 21(1), 1220-1224 (2011).

16. G. Dehaene-Lambertz, S. Dehaene, and L. Hertz-Pannier, "Functional neuroimaging of speech perception in infants," Science 298(5600), 2013-2015 (2002).

17. S. E. Fox et al., "Neural processing of facial identity and emotion in infants at high-risk for autism spectrum disorders," Front. Hum. Neurosci. 7(89), 1-18 (2013).

18. Y. Minagawa-Kawai et al., "Optical brain imaging reveals general auditory and language-specific processing in early infant development," Cereb. Cortex 21(2), 254-261 (2011).

19. S. Lloyd-Fox et al., "Selective cortical mapping of biological motion processing in young infants," J. Cogn. Neurosci. 23(9), 2521-2532 (2011).

20. D. Tsuzuki and I. Dan, "Spatial registration for functional near-infrared spectroscopy: from channel position on the scalp to cortical location in individual and group analyses," Neuroimage 85(1), 92-103 (2014).

21. D. Tsuzuki et al., "Stable and convenient spatial registration of standalone NIRS data through anchor-based probabilistic registration," Neurosci. Res. 72(2), 163-171 (2012).

22. D. Tsuzuki et al., "Virtual spatial registration of stand-alone fNIRS data to MNI space," Neuroimage 34(4), 1506-1518 (2007).

23. A. K. Singh et al., "Spatial registration of multichannel multi-subject fNIRS data to MNI space without MRI," Neuroimage 27(4), 842851 (2005).

24. H. Watanabe et al., "Effect of auditory input on activations in infant diverse cortical regions during audiovisual processing," Hum. Brain Mapp. 34(3), 543-565 (2013).

25. M. Matsui et al., "Referential framework for transcranial anatomical correspondence for fNIRS based on manually traced sulci and gyri of an infant brain," Neurosci. Res. 80, 55-68 (2014).

26. F. Shi et al., "Infant brain atlases from neonates to 1-and 2-year-olds," PLoS One 6(4), e18746 (2011).

27. I. S. Gousias et al., "Automatic segmentation of brain MRIs of 2-yearolds into 83 regions of interest," Neuroimage 40(2), 672-684 (2008).
28. P. Fillmore et al., "Stereotaxic MRI brain atlases for infants from 3 to 12 months," submitted.

29. M. C. Phillips et al., "A stereotaxic MRI brain atlas for infant participants," presented at the Society for Research in Child Development, Seattle (2013).

30. J. E. Richards, M. Stevens, and A. Connington, "A stereotaxic MRI brain atlas for infant participants," Dev. Med. Child Neurol. 54 (Supp. 2), 9-10 (2012).

31. D. W. Shattuck et al., "Construction of a 3D probabilistic atlas of human cortical structures," Neuroimage 39(3), 1064-1080 (2008).

32. A. Hammers et al., "Three-dimensional maximum probability atlas of the human brain, with particular reference to the temporal lobe," Hum. Brain Mapp. 19(4), 224-247 (2003).

33. R. A. Heckemann et al., "Automatic anatomical brain MRI segmentation combining label propagation and decision fusion," Neuroimage $\mathbf{3 3}$ (1), 115-126 (2006).

34. R. A. Heckemann et al., "Information extraction from medical images: developing an e-Science application based on the Globus toolkit," in Proc. 2nd UK E-Sci. Hands Meet., EPSRC, Nottingham, UK (2003).

35. R. Bhopal, "Glossary of terms relating to ethnicity and race: for reflection and debate," J. Epidemiol. Community Health 58(6), 441-445 (2004).

36. S. Lloyd-Fox et al., "Reduced neural sensitivity to social stimuli in infants at risk for autism," Proc. R. Soc. B Biol. Sci. 280(1758), 20123026 (2013).

37. N. L. Everdell et al., "A frequency multiplexed near-infrared topography system for imaging functional activation in the brain," Rev. Sci. Instrum. 76(9), 093705 (2005).

38. A. Simmons, E. Moore, and S. C. Williams, "Quality control for functional MRI using automated data analysis and Shewhart charting," Magn. Reson. Med. 41(9), 1274-1278 (1999).

39. C. E. Sanchez, J. E. Richards, and C. R. Almli, "Age-specific MRI templates for pediatric neuroimaging," Dev. Neuropsychol. 37(5), 379-399 (2012).

40. C. E. Sanchez, J. E. Richards, and C. R. Almli, "Neurodevelopmental MRI brain templates for children from 2 weeks to 4 years of age," Dev. Psychobiol. 54(1), 77-91 (2012).

41. J. E. Richards, "Neurodevelopmental MRI database," http ://jerlab .psych.sc.edu/NeurodevelopmentalMRIDatabase (1 August 2014).

42. V. Fonov et al., "Unbiased average age-appropriate atlases for pediatric studies," Neuroimage 54(1), 313-327 (2011).

43. U. Yoon et al., "The effect of template choice on morphometric analysis of pediatric brain data," Neuroimage 45(3), 769-777 (2009).

44. B. B. Avants et al., "Symmetric diffeomorphic image registration with cross-correlation: evaluating automated labeling of elderly and neurodegenerative brain," Med. Image Anal. 12(1), 26-41 (2008).

45. M. Jenkinson, M. Pechaud, and S. Smith, "BET2: MR-based estimation of brain, skull and scalp surfaces," in Eleventh Annual Meeting of the Organization for Human Brain Mapping, Toronto, Canada, Vol. 17 (2005).

46. S. M. Smith et al., "Advances in functional and structural MR image analysis and implementation as FSL," Neuroimage 23(Supp 1), S208-S219 (2004).

47. M. W. Woolrich et al., "Bayesian analysis of neuroimaging data in FSL," Neuroimage 45(1), S173-S186 (2009).

48. J. Hill et al., "A surface-based analysis of hemispheric asymmetries and folding of cerebral cortex in term-born human infants," J. Neurosci. Off. J. Soc. Neurosci. 30(6), 2268-2276 (2010).

49. T. Rohlfing et al., "The SRI24 multichannel atlas of normal adult human brain structure," Hum. Brain Mapp. 31(5), 798-819 (2010).

50. J. E. Richards, "Scalp surface locations and underlying cortical areas in infants," unpublished manuscript (2013).

51. J. Talairach and P. Tournoux, Co-Planar Stereotaxic Atlas of the Human Brain. 3-Dimensional Proportional System: An Approach to Cerebral Imaging, Thieme, New York (1988).

52. C. Rorden, H.-O. Karnath, and L. Bonilha, "Improving lesion-symptom mapping," J. Cogn. Neurosci. 19(7), 1081-1088 (2007).

53. J. E. Richards, "Cortical sources of ERP in prosaccade and antisaccade eye movements using realistic source models," Front. Syst. Neurosci. 7 (27), 1-20 (2013). 
54. V. Jurcak, D. Tsuzuki, and I. Dan, "10/20, 10/10, and 10/5 systems revisited: their validity as relative head-surface-based positioning systems," Neuroimage 34(4), 1600-1611 (2007).

55. M. Okamoto and I. Dan, "Automated cortical projection of head-surface locations for transcranial functional brain mapping," Neuroimage 26(1), 18-28 (2005).

56. F. Van Overwalle and K. Baetens, "Understanding others' actions and goals by mirror and mentalizing systems: a meta-analysis," Neuroimage 48(3), 564-584 (2009).

57. A. Cristia et al., "An online database of infant functional near infrared spectroscopy studies: a community-augmented systematic review," PLoS One 8(3), e58906 (2013).

58. A. Cristia, "DFIfNIRS," https://sites.google.com/site/dbifnirs/ (26 February 2014)

59. T. Correia et al., "Three-dimensional optical topography of brain activity in infants watching videos of human movement," Phys. Med. Biol. 57(5), 1135-1146 (2012).

60. M. D. Papademetriou et al., "Optical topography image reconstruction of brain activation in Gambian infants," in Oxygen transport tissue $X X X V$, S. Van Huffel et al., Eds., Vol. 789, pp. 455-461, Springer, New York (2013).

Sarah Lloyd-Fox is a research fellow at the Centre for Brain and Cognitive Development, Birkbeck, University of London. Her work focuses on the use of fNIRS to investigate the developing brain in infancy, focusing on social cognition and developmental neuroscience. Her work also examines brain function in infants at risk of compromised development due to neurodevelopmental disorders and most recently undernutrition. This has led to the novel application of fNIRS in resource-poor countries.

John E. Richards received his PhD degree from UCLA and is currently in the Department of Psychology and Institute for Mind and Brain at the University of South Carolina. His research examines the relationship between the development of attention in infants and brain development. He also studies attention and face processing in infants with neurodevelopmental disorders.
Anna Blasi is a research fellow at the Centre for Brain and Cognitive Development, Birkbeck, University of London. Her research interests are centered on functional aspects of human physiology. Her research career started with models of the cardiovascular system and the effects of disease. Through her work at UCL, KCL, and Birkbeck, her research interests have shifted toward the use of functional imaging (fNIRS, fMRI) to study brain function and neurocognitive development in early infancy.

Declan G. M. Murphy holds the Mortimer D. Sackler Chair in translational neurodevelopment and leads the EU IMI AIMS network on autism. His research mainly focuses on the biological determinants of brain development and how abnormalities in this process lead to neuropsychiatric disorders. He undertook his clinical psychiatry training at the Maudsley Hospital, and his research training at the Institute of Psychiatry (King's College, London) and the National Institute on Aging (National Institutes of Health, USA).

Clare E. Elwell is a professor of medical physics in the Department of Medical Physics and Bioengineering at UCL. She leads the near-infrared spectroscopy (NIRS) research group developing novel optical systems for monitoring and imaging the human body and brain. Her research projects include studies of autism, acute brain injury, sports performance, migraine, malaria, depression, and, most recently, the effects of malnutrition on brain development with the first infant functional brain imaging study in Africa.

Mark H. Johnson is a Medical Research Council scientific program leader and director of the Centre for Brain and Cognitive Development, Birkbeck (University of London). He is also a fellow of the British Academy and the Cognitive Science Society. He has published over 250 papers and 10 books on brain and cognitive development in human infants and other species. His laboratory currently focuses on typical and atypical functional brain development during infancy and childhood. 